\title{
Casting Simulation and Prediction of Shrinkage Cavities
}

\author{
Jabur AS $^{1 *}$ and Kushnaw FM $^{2}$ \\ ${ }^{1}$ University of Basrah, Iraq \\ ${ }^{2}$ University of Technology, Baghdad, Iraq
}

\begin{abstract}
This work aims to predict the shrinkage defects in Al-Si castings by determination the suitable parameters and techniques which can be applied in the casting simulation system. Also, it aims to specify the role of silicon content in amount, morphology, and distribution of these defects. The Numerical solution has been carried out using an explicit 3-D finite difference method for the given system of the casting and a mold. Additionally, an experimental casting of the studied samples was achieved. It was found that the shrinkage porosities increased with increasing the silicon content up to $7 \%$, so at this peak, they spread in all cast regions and cannot be predicted. The low silicon alloys suffered from only the cavities defects that can be predicted by mapping the solidus time contours. Finally, it was concluded that the critical temperature gradient value of the porosities development in the eutectic (Al-12\%Si) alloys was $1.3^{\circ} \mathrm{C} / \mathrm{cm}$.
\end{abstract}

Keywords: Simulation; Al-Si alloys; Casting structures; Numerical analysis; Shrinkage defects

\section{Introduction}

Producing sound aluminum-silicon castings is one of great economic significance to the foundry industry; however, shrinkage defects are the main categories of casting defects which take many forms; shrinkage cavity, porosity and surface sink. Such defects have a negative economic impact on casting production; their consequences range from high rework costs to casting rejection as reported by investigators [1]. During recent years, the application of some popular commercial software's as computer simulation tools has become widely accepted within the foundry industry [2]. The application of casting simulation has been most beneficial, for avoiding shrinkage scrap, by predication of shrinkage defects without having to discover them in the foundry through the usual trial and error process [3], which can be very tedious, time consuming, and expensive [4]. One of the methods to describe solidification modeling is the heat transfer model, which solves the energy equation [5]. There have been few studies to model the effect of solidification shrinkage from different perspectives. Trovant and Argyropoulos [6] proposed an algorithm to account for shrinkage and consequently determine the shrinkage profile resulting from phase and density change. Only the energy equation is solved in the domain and the effects of solidification shrinkage are imposed through the proposed algorithms. Bride [7] presented a model that accounts for shrinkage during the directional solidification or dendritic binary alloy under the assumption that the densities of liquid and solid phase are different but constant. Also, Kim and Ro [8] performed a study on shrinkage formation during solidification of a material in a twodimensional rectangular cavity. Anderaus and Delliisola [9] proposed a 2-D finite difference model to describe the influence of solidification shrinkage on the final shape of cast ingot. Paszndiden-Fard et.al [10] develop a numerical method to simulate the solidification shrinkage of the solidifying liquid [11-13].

The aim of this work is to find the suitable criteria or methods that can be used in prediction the shrinkage defects. Also, it aims to study the effect of silicon content on the morphology, quantity, and distribution of the shrinkage defects in the Al-Si castings.

\section{Mathematical Model}

\section{Metal region}

The variation of temperature $T$ with time could be described by solving the Fourier heat equation, which is generally written as:

$$
\rho C_{p}\left(\frac{\partial T}{\partial \tau}\right)=K\left[\left(\frac{\partial^{2} T}{\partial x^{2}}\right)+\left(\frac{\partial^{2} T}{\partial y^{2}}\right)+\left(\frac{\partial^{2} T}{\partial z^{2}}\right)\right]+\dot{q}
$$

Where convection is neglected. The density denotes as $\rho$, specific heat of casting as $C_{p}$ and the term $\rho C_{p}(\partial T / \partial \tau)$ is the transient term where, $\tau$ is a time step. In the right side the three terms represent the heat conduction in three-direction with thermal conductivity $K$. A source term $\dot{q}$ is an internal heat generation for solidification under equilibrium condition. This term can be described as:

$$
\dot{q}=\rho L \frac{\partial f_{\mathrm{s}}}{\partial \tau}=\rho L \frac{d f_{\mathrm{s}}}{d T} \frac{d T}{d \tau}
$$

Where $L$ denotes latent heat distributed over the solidification range, so it is a function of the solid fraction $\left(f_{s}\right)$ as confirmed by Poirier and Salcudean [11].

Thermo physical properties in the mushy region will depend upon the amount of liquid and solid (i.e. solid fraction) and can be calculated as following:

$$
P_{T}=f_{s(T)} P_{s o l}+\left(1-f_{s(T)}\right) P_{l i q}
$$

Where $f_{s(T)}$ are the solid fraction at $T$ and $P_{\text {sol }}$ and $P_{\text {liq }}$ are values of the property at solidus and liquidus temperatures, respectively.

If the alloy has very narrow solidification interval or it is a pure metal or a eutectic, $T_{S}$ will be close to or equal to $T_{L}$. In this case an artificial temperature interval has to be assumed. This temperature *Corresponding author: JaburAS, University of Basrah, Iraq, Tel: 9647802443867 ;
E-mail: ednan909@yahoo.com

Received September 08, 2017; Accepted October 13, 2017; Published October 23, 2017

Citation: Jabur AS, Kushnaw FM (2017) Casting Simulation and Prediction of Shrinkage Cavities. J Appl Computat Math 6: 371. doi: 10.4172/2168-9679.1000371

Copyright: @ 2017 Jabur AS, et al. This is an open-access article distributed under the terms of the Creative Commons Attribution License, which permits unrestricted use, distribution, and reproduction in any medium, provided the original author and source are credited. 
interval should be as small as possible to be near the real material behavior.

$$
f_{l}=\left(\frac{T-T_{s}}{T_{l}-T_{s}}\right)
$$

\section{Mold region}

For heat conduction in the mold, the energy equation is:

$$
\rho_{m} C_{p m}\left(\frac{\partial T_{m}}{\partial \tau}\right)=K_{m}\left[\left(\frac{\partial^{2} T_{m}}{\partial x^{2}}\right)+\left(\frac{\partial^{2} T_{m}}{\partial y^{2}}\right)+\left(\frac{\partial^{2} T_{m}}{\partial z^{2}}\right)\right]
$$

the subscript $m$ is denoted the mold and $K_{m}$ is the mold thermal conductivity.

\section{Boundary conditions}

On the outer surface, heat transfer is done by convection as following:

$$
-K_{m} \frac{\partial T_{m}}{\partial x}=h\left(T_{m}-T_{a}\right)
$$

where $T_{m}$ is the temperature of outer surface of mold and $T_{a}$ is ambient temperature.

\section{Assumptions made}

The following assumptions were made for the formulation of the problem:

1. Mold is filled instantaneously.

2. Phase transformation proceeds in one direction: liquid to solid.

3. No convection is present in the liquid metal.

4. Thermal properties of the metal casting for liquid and solid phase are different but constant.

The density considered variable with temperature.

\section{Numerical solution}

After discretization of the whole domain and using the forward difference for the time derivative and the central difference for the spatial second derivative, the approximation resulting in :

$$
T_{i, j, k}^{m+1}=T_{i, j, k}^{m}+\frac{K \Delta \tau}{\rho C_{p}}\left[\frac{T_{i-1, j k}^{m}-2 T_{i, j, k}^{m}+T_{i+1, j, k}^{m}}{\Delta x^{2}}+\frac{T_{i, j-1, k}^{m}-2 T_{i, j, j}^{m}+T_{i, j+1, k}^{m}}{\Delta y^{2}}+\frac{T_{i, j, k-1}^{m}-2 T_{i, j, k}^{m}+T_{i, j, k+1}^{m}}{\Delta z^{2}}\right]
$$

Equation (14) is called the Forward Time, Centered Space or FTCS approximation to the heat equation. It can be reduced as:

$$
T_{i, j, k}^{m+1}=(1-6 F) T_{i, j, k}^{m}+F\left(T_{i-1, j, k}^{m}+T_{i+1, j, k}^{m}\right)+F\left(T_{i, j-1, k}^{m}+T_{i, j+1, k}^{m}\right)+F\left(T_{i, j, k-1}^{m}+T_{i, j, k+1}^{m}\right)
$$

The FTCS scheme is easy to implement because the values can be updated independently of each other. The entire solution is contained in two loops: an outer loop over all time steps and an inner loop over all interior nodes. Notice that The FTCS can yield unstable solutions that oscillate and grow if $\Delta \tau$ is too large. According to Jaluria [12], stable solutions with the FTCS scheme are only obtained if;

$$
F=\frac{K \Delta \tau}{\rho C_{P}(\Delta x)^{2}} \leq \frac{1}{6}
$$

\section{Computer programming}

A computer program has been built in using FORTRAN 90, to carry out the numerical solution, on a personal computer. The basic structure of this program is shown in Figure 1. Dimensions of casting are illustrated in Figures 2 and 3.

\section{Experimental Procedure}

After melting the aluminum alloys in a gas furnace and degassing with a $\mathrm{C}_{2} \mathrm{Cl}_{6}$ tablets, the molten metal were poured in sand molds. Seven straight bar samples (Figure 2) with different dimensions were prepared to study the effect of silicon percentage on the shrinkage defects. Also, a rectangular sample (Figure 3) with fin was prepared to evaluate the efficiency of the program in prediction the cavities location. Details of these samples were contained in Table 1.

\section{Results and Discussion}

\section{Verification of the model}

In order to check the program, numerical simulation results

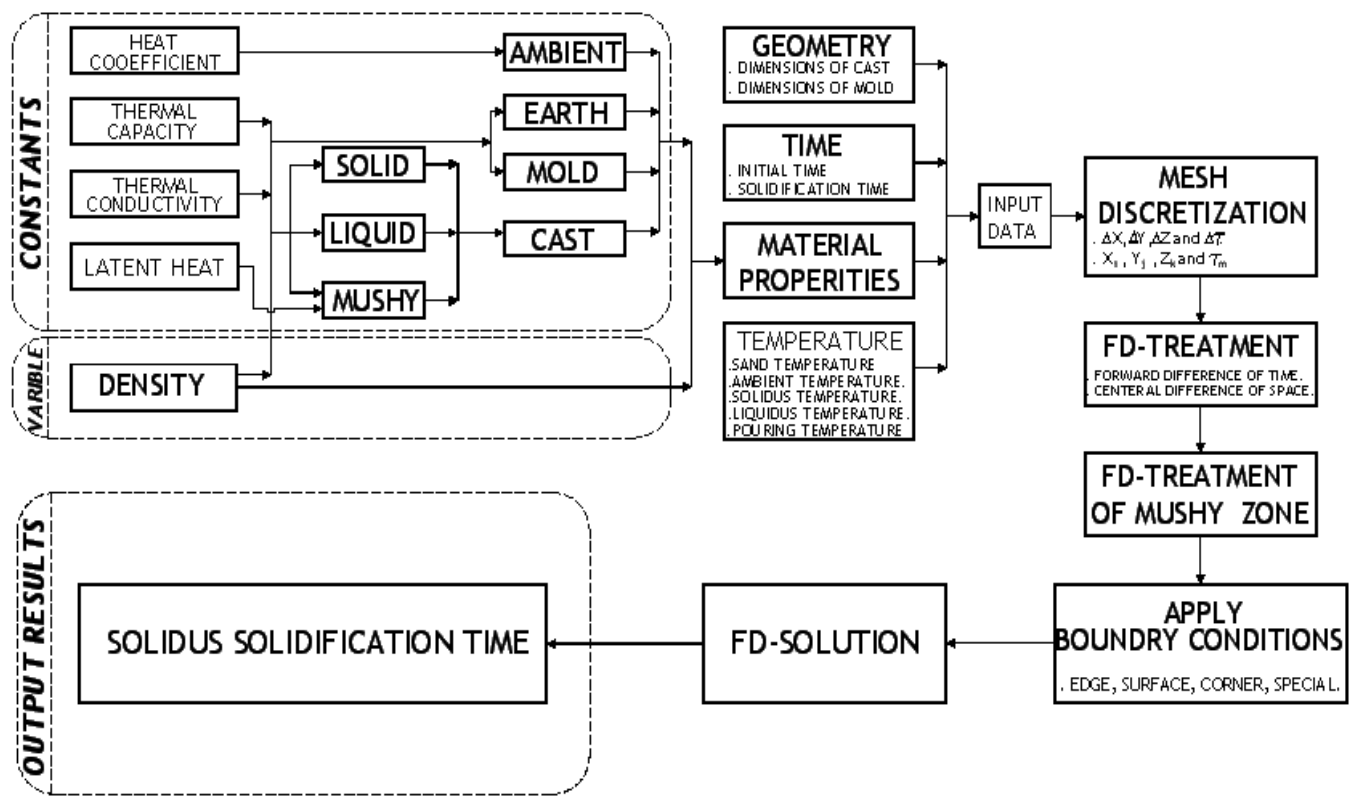

Figure 1: Basic Structure of FD- program. 


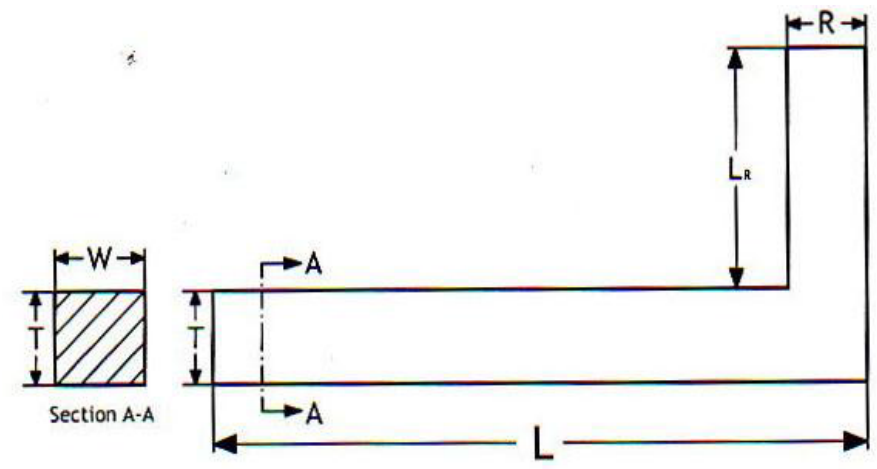

Figure 2: Straight bar samples.

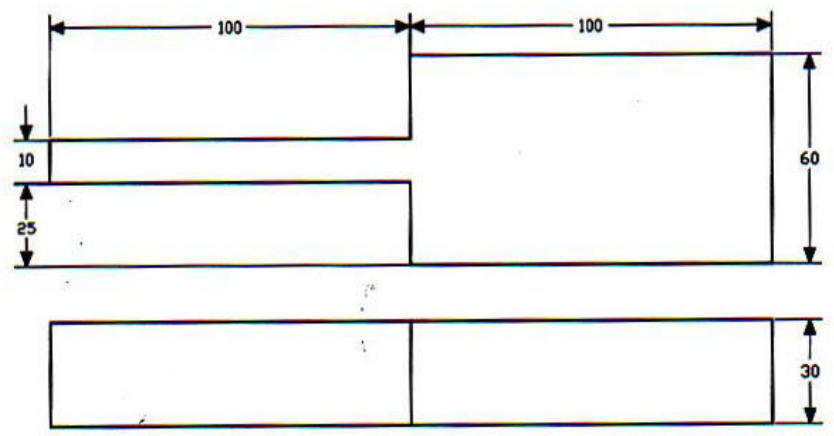

Figure 3: Rectangular Samples.

\begin{tabular}{|c|c|c|c|c|c|c|c|c|c|}
\hline Sample code & Composition & $\begin{array}{l}\text { Cast length } \\
\text { mm }\end{array}$ & $\begin{array}{l}\text { Cast width } \\
\text { mm }\end{array}$ & $\begin{array}{l}\text { Cast thickness } \\
\text { mm }\end{array}$ & $\begin{array}{l}\text { Feeder } \\
\text { length } \mathrm{mm}\end{array}$ & $\begin{array}{l}\text { Feeder Diameter } \\
\text { mm }\end{array}$ & $\begin{array}{c}\text { Pouring temp. } \\
{ }^{\circ} \mathrm{C}\end{array}$ & $\begin{array}{c}\text { Solidus temp. } \\
{ }^{\circ} \mathrm{C}\end{array}$ & $\begin{array}{l}\text { Liquius temp. } \\
{ }^{\circ} \mathrm{C}\end{array}$ \\
\hline 1 & $\mathrm{Al}-12 \% \mathrm{Si}$ & 500 & 50 & 30 & 145 & 45 & 610 & 542 & 573 \\
\hline 2 & Al-12\% Si 5 & 520 & 35 & 30 & 145 & 45 & 610 & 542 & 573 \\
\hline 3 & $\mathrm{Al}-12 \% \mathrm{Si}$ & 480 & 70 & 30 & 145 & 45 & 610 & 542 & 573 \\
\hline 4 & Al-7\% Si 4 & 470 & 45 & 30 & 145 & 45 & 650 & 567 & 614 \\
\hline 5 & $\mathrm{Al}-0.85 \% \mathrm{Si}$ & 480 & 45 & 30 & 145 & 45 & 690 & 643 & 648 \\
\hline 6 & Pure Al & 500 & 50 & 30 & 145 & 45 & 705 & 660 & 660 \\
\hline 7 & Pure Al & 100 & 60 & 30 & - & 45 & 705 & 660 & 660 \\
\hline
\end{tabular}

Table 1: Dimension of the casting samples.

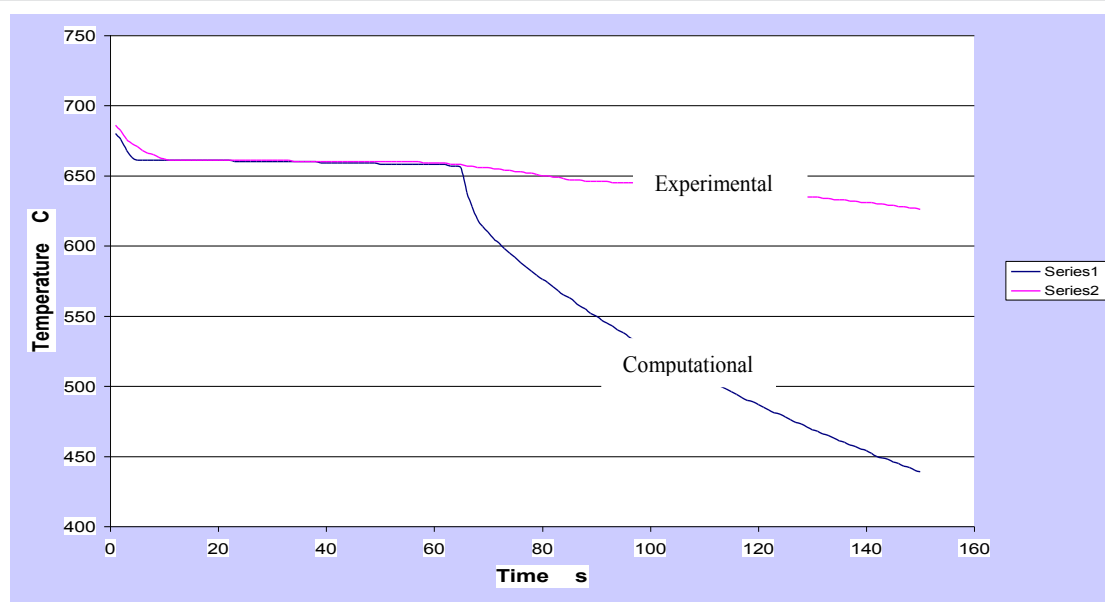

Figure 4: Comparison Between Simulated And Experimental Results At The Centre Of The Cast (1).

were compared with experimental data. It conducted by inserting thermocouple in the cast Sample (1). Figure 4 shows the simulated and experimental cooling curves at cast center position. It is obvious that the slope of the simulated curve after solidification is smaller than 
experimental one which may be due to the effect of solid contraction and consequently the air gap development. But, at the interval between the liquidus and solidus temperature (local solidification time), It represents a quite similar between them.

\section{The effect of Si percentage on the shrinkage morphology}

Four samples with different silicon percentages are arranged in Table 2. It contains the shrinkage morphologies extracted from Figure 5 shows that, the pure aluminum (sample 6), suffered from only one type of shrinkage defects. Feeder tends to pipe deeply; the pipe is smooth and geometric in shape, with the absence of any type of shrinkage porosities as shown in Figures 6 and 7. This can be attributed to the plane front solidification behavior of pure alloys. Two reasons to the severe extent of pipe in these samples. First, the high solidification contraction of pure aluminum (7.14\%). Second, the absence of the other types of shrinkages leads to accumulation in the pipe only.

Sample (5) of low silicon content $(0.85 \%)$ had approximately the same shrinkage behavior as the pure aluminum samples. Here the pipe is less regular and the end is ragged as shown in Figure $5 c$.
Sample (4) of 7\% silicon exhibited light pipe and a dispersed porosity in all cast regions, as shown in Figure $5 \mathrm{~b}$ and Figure 6. This is explained by the long freezing range of this composition which offered a high constitutional super cooling and an effective dendritic solidification behavior and consequently a limited feeding to the interdendritic shrinkage regions so that a nucleation of porosities enhanced. On the other hand, the reason of the presence of small pipe in comparison with the previous alloys is firstly; that the total contraction percentage of $\mathrm{Al}-\mathrm{Si}$ alloys decreases with increasing the silicon content. Secondly; the total contraction here shared between the pipe and wide dispersed porosities.

Sample (1) of $12 \%$ silicon (Figure 5a and Figure 8) exhibited medium size pipe and localized porosities in the centerline. The short freezing range of this alloy produced a simple constitutional super cooling resulted in cellular or light dendritic freezing mode for the eutectic layers or for the aluminum grains which contain the porosities in between. The pipe of this sample is smaller than that of sample (6) because the total contraction percentage of this alloy is $3.8 \%$; also it is larger than that of sample (4) because the porosities here are localized in the centerline only.

\begin{tabular}{|c|c|c|c|c|}
\hline Sample Code & \% Si & Pipe & Centerline porosities & Dispersed porosities \\
\hline $\mathbf{1}$ & 12 & Medium & Present & Nil \\
\hline $\mathbf{4}$ & 7 & Light & Nil & Severe \\
\hline $\mathbf{5}$ & 0.85 & severe & Nil & Nil \\
\hline
\end{tabular}

Table 2: Shrinkage Defect Morphologies Of The Casting Samples.
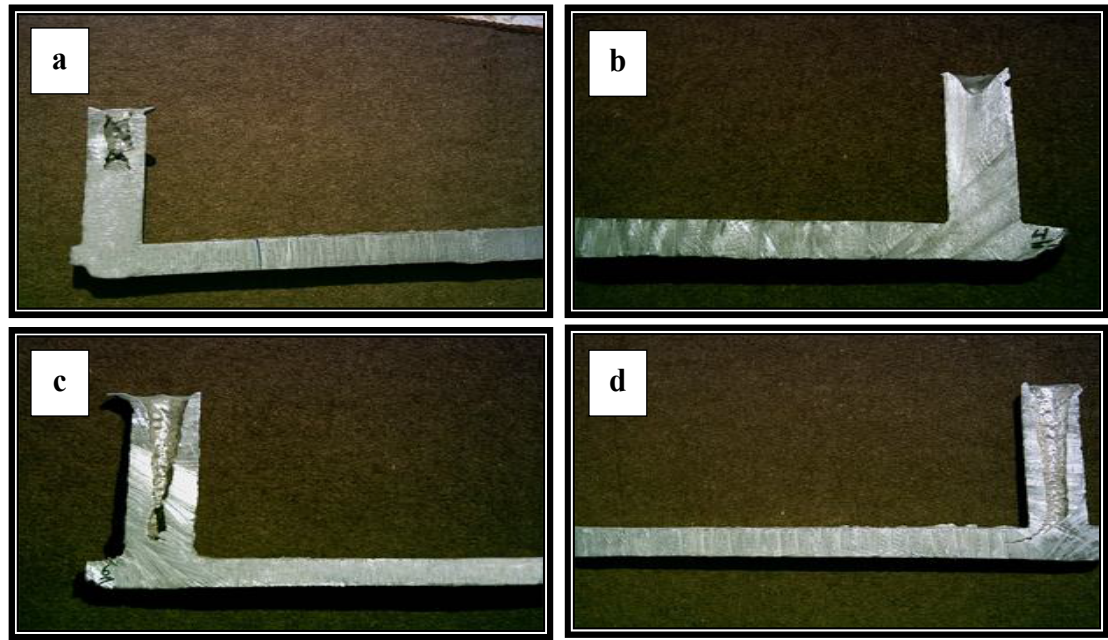

Figure 5: Photographs of A-Sample (1), B-Sample (4), C-Sample (5), And D-Sample (6) Sections, Which Illustrate The Shrinkage Defect Morphologies.

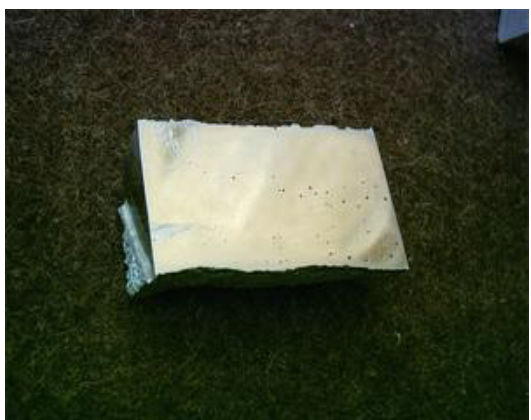

Figure 6: A Photograph of Polished Section of Sample (4) Shows The Dispersed Porosities. 
Citation: Jabur AS, Kushnaw FM (2017) Casting Simulation and Prediction of Shrinkage Cavities. J Appl Computat Math 6: 371. doi: 10.4172/21689679.1000371

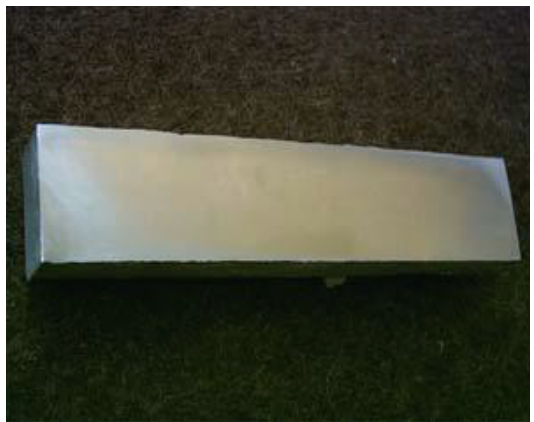

Figure 7: A Photograph of Polished Section of Sample (6) Shows The Porosity Free Surface.
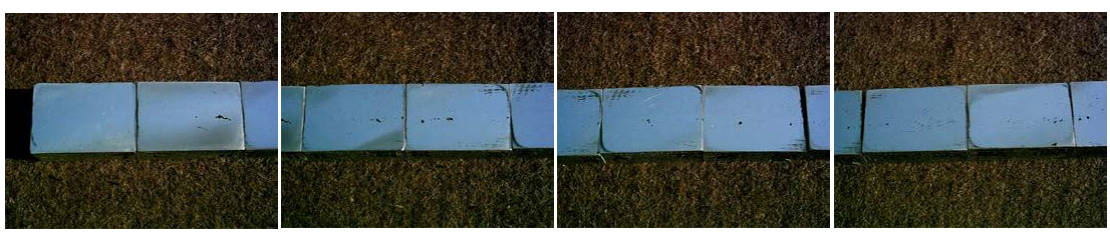

Figure 8: A Photograph of Polished Section of Sample (1) Shows The Centerline Porosities.

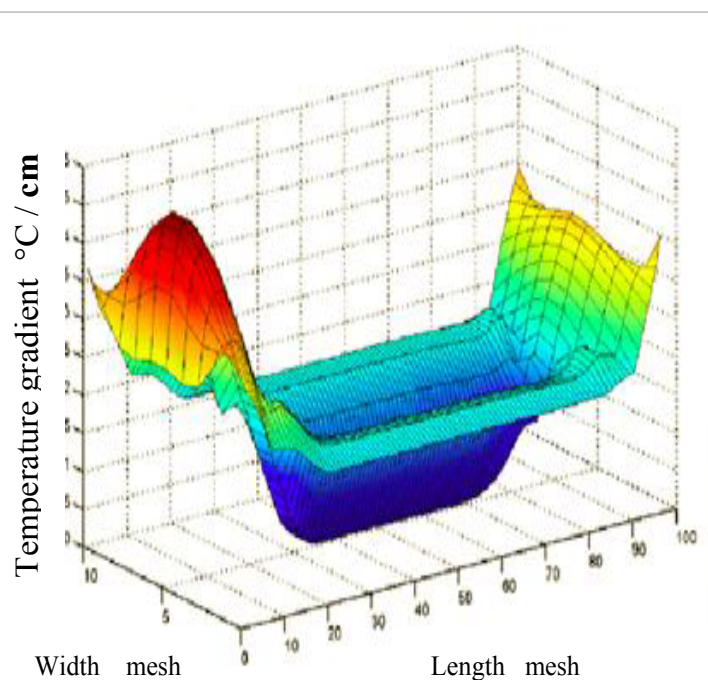

${ }^{\circ} \mathrm{C} / \mathrm{cm}$

Figure 9: The Gradient variation over the mid surface of sample (1).

\section{Simulation results}

\section{Aluminum - 12\% Silicon}

Figures 9-11 shows the distribution of the temperature gradient through (x-y) mid surface of samples (1,2 and 3) respectively. The temperature gradients near the wall area are high due to rapid wall chilling.

Figure 12 contains the values of the porosity length extensions in these samples in addition to the gradient values at the starting and ending points. The region of shrinkage coincides with the region of shallow temperature gradient (center line shrinkage). In fact, all of the shrinkage porosities were found within the region of calculated temperature gradient of lower than $1.3^{\circ} \mathrm{C} / \mathrm{cm}$. Therefore, $1.3^{\circ} \mathrm{C} / \mathrm{cm}$ was tentatively taken as the critical value of shrinkage occurrence. The equivalence of these values at the two points for a one sample and

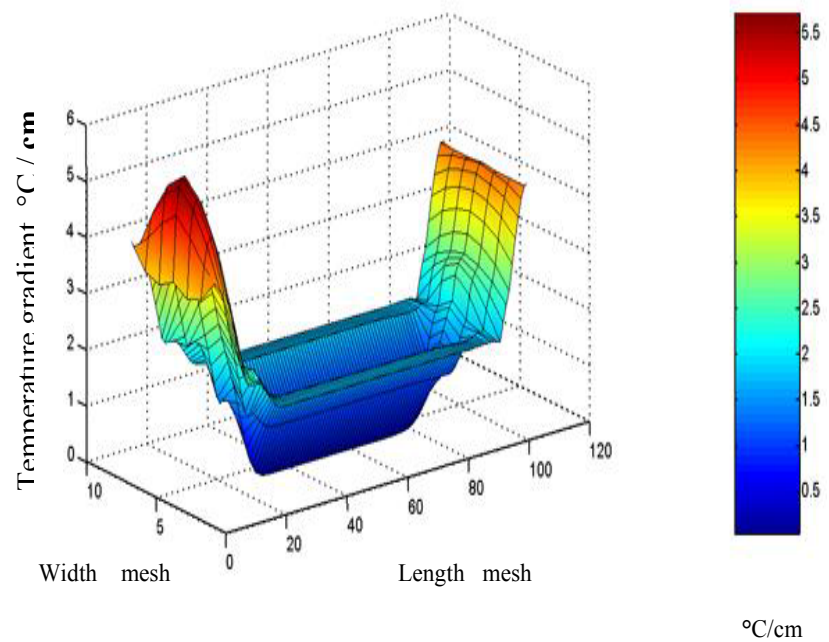

Figure 10: The Gradient variation over the mid surface of sample (2).

between the three different dimensions samples gives a good support for this proposal. Niyama et al. [13] reports the values of the critical temperature gradient; 2 to $3^{\circ} \mathrm{C} / \mathrm{cm}$ for the steel bars. The difference between Niyama values and ours is not surprising because the values must depend on casting type, alloy composition. Niyama did not take into account the change in thermal properties for liquid and solid. While Niyama's criterion works well for low carbon steel that has very small difference between solid and liquid properties that makes its application by many nonferrous foundries is questionable because they have large diverge of thermal properties between solid and liquid metal.

\section{Aluminum - 7\% silicon}

Figure $5 \mathrm{~b}$ and Figure 6 include photographs of the polished section of sample (4), which show a wide dispersed of shrinkage porosities through the sample section without any concentration in a specified region. Figure 13 illustrates the distribution of the temperature 


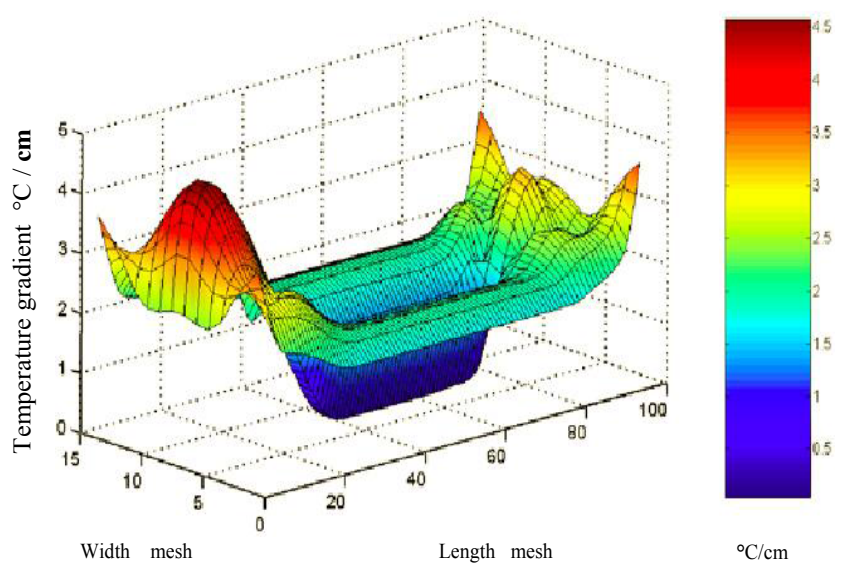

Figure 11: The Gradient variation over the mid surface of sample (3).

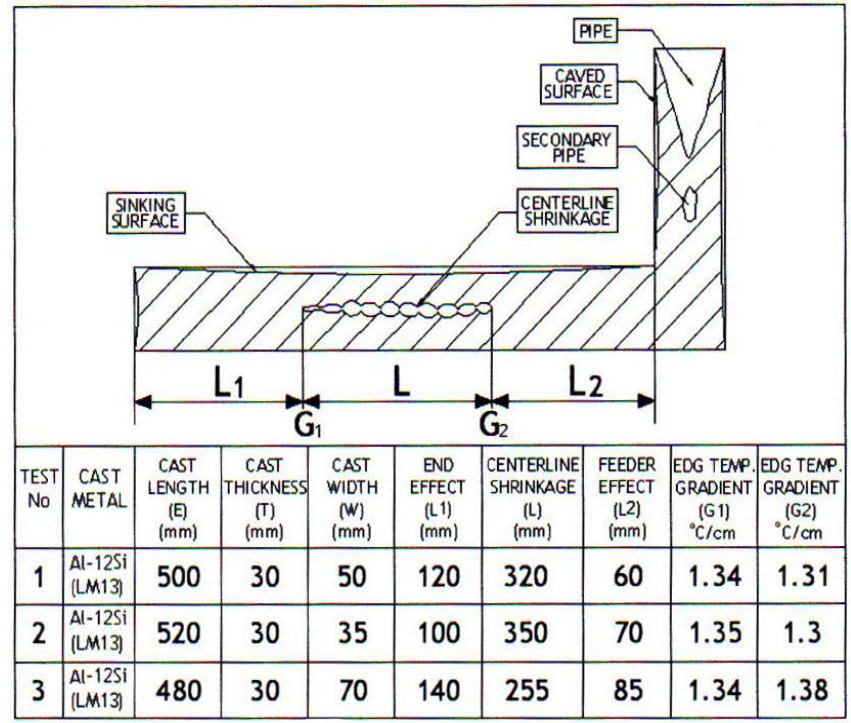

Figure 12: Values of Critical Temperature Gradients Which Is Gained From Simulation.

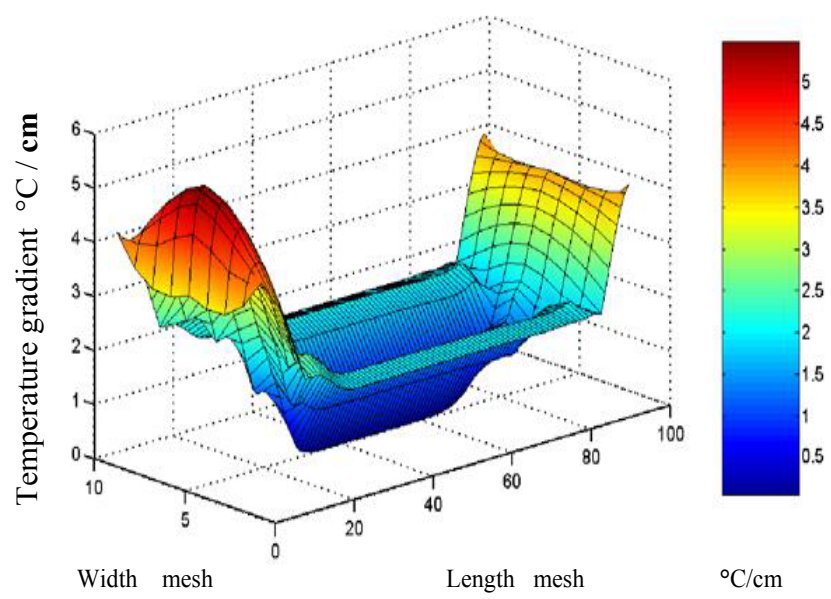

Figure 13: The Gradient variation over the mid surface of sample (4).

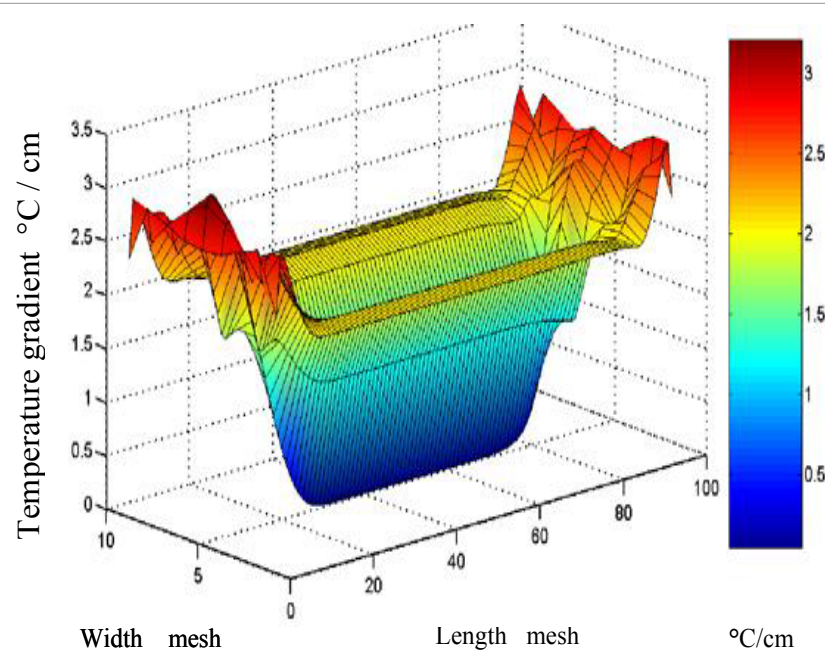

Figure 14: The Gradient variation over the mid surface of sample (5).

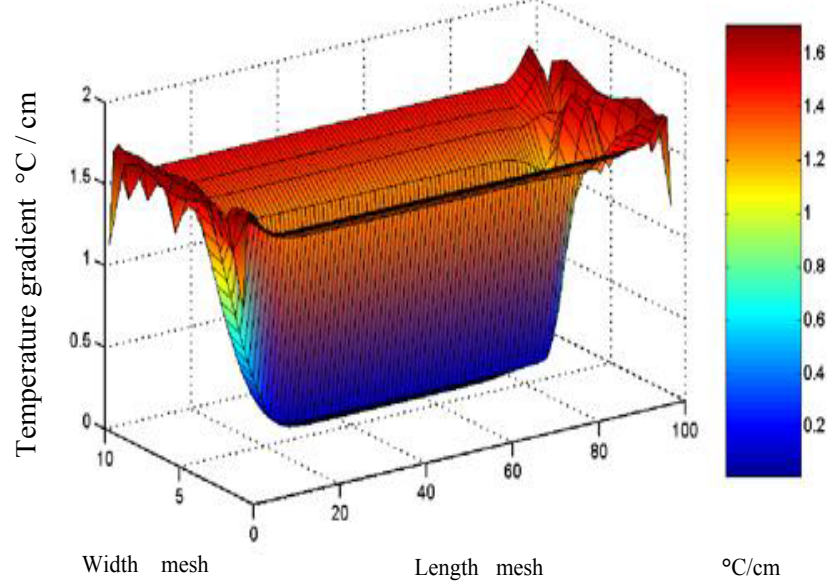

Figure 15: The Gradient variation over the mid surface of sample (6)

gradient through the mid surface $(x-y)$. It clarifies that; the shrinkage porosities distribution in this silicon concentration does not depend on this parameter values, and they cannot be predicted with this tool.

\section{Aluminum - 0.85\% silicon}

Figure 14 shows the distribution of the temperature gradient through the mid surface ( $x-y)$ for sample (5). Also, the gradient decreased for very low values and did not cause a development of porosities. Therefore, their solidification behavior resemble to the pure aluminum.

\section{The pure aluminum}

The first extension of the modeling is the evaluation of the temperature gradient over the mid surface $(x-y)$ of the pure aluminum (sample 6) as in Figure 15. In general, the gradients are highest at the casting edges, but decrease with moving toward the casting center. These samples are free from the porosities in a different manner than steel, i.e. the development of the porosities depends on the decreasing of the temperature gradient under a critical value as the work of Niyama et al. [13]. Consequently, the development of the porosities in pure aluminum did not depend on the gradient values because 


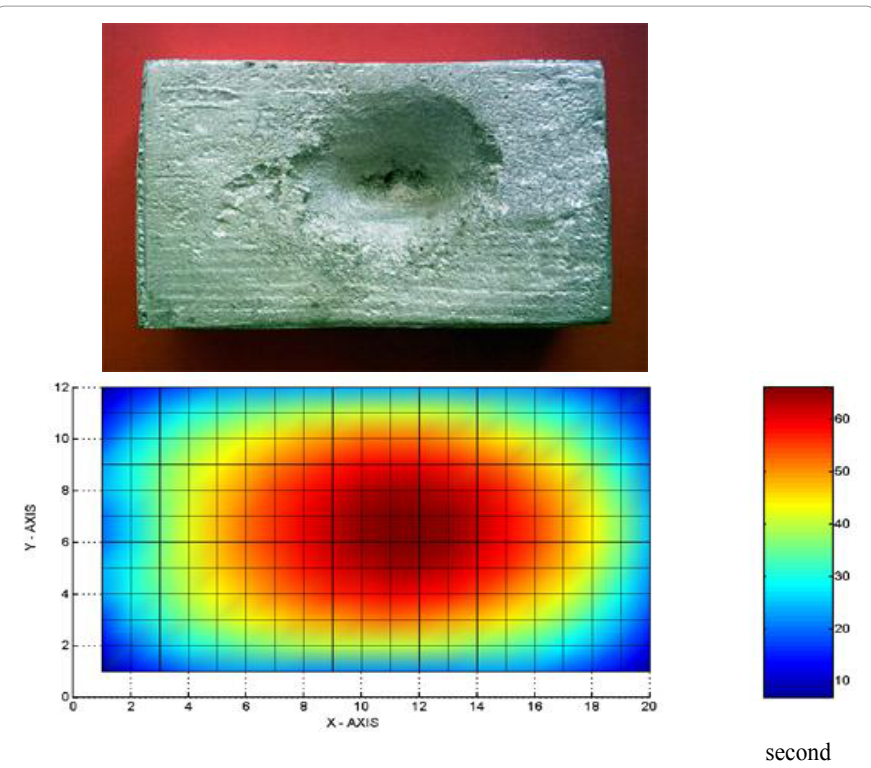

Figure 16: The solidus time contours over the mid surface of sample (7).

the solidification front type (planar) has the predominant role in this manner and the gradient has no role.

The solidification time can be used in the cavities prediction .The appearance of a closed loop in the solidification time contours is the suitable criterion for this type of shrinkage. For checking the efficiency of the present simulation program with employing the solidification time criterion in prediction the cavities position, a rectangular sample designed to produce an internal cavity was tested (sample 7). Figure 16 includes a comparison between the sliced sample (to identify the position of the real cavity) and a simulated graph of the solidus time contours over the same sliced surface. There is a good agreement between them that make this criterion a good tool in cavities prediction and a successful of the present computer program in the simulation function to predict the hot spot and consequently the cavity.

\section{Conclusions}

From the present work, the main conclusions can be summarized as:

1. The shrinkage porosities increase with increasing the Si content up to $7 \%$, so at this peak, they spread in all cast regions and cannot be predicted.
2. The low $\mathrm{Si}$ and pure Al-alloys castings suffered from the shrinkage cavities defects only which can be predicted by mapping the solidus time contours.

3. The critical temperature gradient value of the shrinkage porosities development in the eutectic $(\mathrm{Al}-12 \% \mathrm{Si})$ alloys was $1.3^{\circ} \mathrm{C} / \mathrm{cm}$.

\section{References}

1. Beckermann C (2002) Modeling of Solidification, in: Purdue Heat Transfer Celebration. April 3-5 University of lowa, pp: 19-22.

2. Rao PP, Chakrayerthi GACSK, Balakrishna B (2011) Application of Casting Simulation for Sand Casting of a Crusher Plate. International Journal of Thermal Technologies.

3. Guharaja S, Noorul HA, Karuppannan KM (2006) Optimization of Green Sand Casting Process Parameters by Using Taguchis Method. The International Journal of Advanced Manufacturing Technology 30: 1040-1048.

4. Jun $\mathrm{HH}$ (2006) Application of the software ProCAST in the casting of solidification simulation. Journal of Materials Science \& Technology 14: 292-295.

5. Chokkalingam B, Mohamed NSS (2009) Analysis of Casting Defect Through Defect Diagnostic Study Approach. Journal of Engineering Annals of the Faculty of Engineering Hunedoara 2: 209-212.

6. Trovant M, Argyropouos SA (1996) Mathematical modeling and experimental measurement of shrinkage in the casting of metals. Canadian Metallurgical Quarterly 35: 77-84.

7. Bride M (1999) Numerical simulation of incompressible flow driven by density variation during phase change. International. Journal for Numerical Methods in Fluids 3.1: 787-800.

8. Kim C, Ro S (1993) Shrinkage Formation during the Solidification Process in an Open Rectangular Cavity. ASME Journal of Heat Transfer 115: 1078-1081.

9. Andreause U, Delliisola F (1996) On Thermo kinematic Analysis of Pipe Shaping in Cast Ingot: A Numerical Simulation via FDM. International Journal of Engineering Science 34: 1349-1367.

10. Paszndiden-Fard M, Chandra S, Mostaghimi J (2002) A Three Dimensional Model of Droplet Impact and Solidification. Journal of Heat Mass Transfer 45: 2229-2242.

11. Poirier D, Salcudean M (1988) On Numerical Methods Used in Mathematical Modeling of Phase Change in Liquid Metals. Journal of Heat Transfer 110: 562-570.

12. Jaluria $Y(1988)$ Computer Methods for Engineering. First education. Allyn \& Bacon.

13. Niyama E, Uchida T, Morikawa M, Saito S (1982) A Method of Shrinkage Predicting and Its Application to Steel Casting Practice. International Foundry Congress 43: 1711-1715. 